\title{
Determination of the Thermodynamic Properties of Water from the Speed of Sound
}

\author{
J P Martin Trusler*1 and Eric W Lemmon ${ }^{2}$
}

1 Department of Chemical Engineering, Imperial College London, London SW7 2AZ, U.K.

2 Applied Chemical and Materials Division, National Institute of Standards and Technology, 325 Broadway, Boulder, CO 80305-3337, U.S.A.

* Corresponding author. Email: m.trusler@imperial.ac.uk

\begin{abstract}
Thermodynamic properties of compressed liquids may be obtained from measurements of the speed of sound by means of thermodynamic integration subject to initial values of density and isobaric specific heat capacity along a single low-pressure isobar. In this paper, we present an analysis of the errors in the derived properties arising from perturbations in both the speedof-sound surface and the initial values. These errors are described in first order by a pair of partial differential equations that we integrate for the example case of water with various scenarios for the errors in the sound speed and the initial values. The analysis shows that errors in either the speed of sound or the initial values of density that are rapidly oscillating functions of temperature have a disproportionately large influence on the derived properties, especially at low temperatures. In view of this, we have obtained a more accurate empirical representation of the recent experimental speed-of-sound data for water [Lin and Trusler, J. Chem. Phys. 136, (2012) 094511] and use this in a new thermodynamic integration to obtain derived properties including density, isobaric heat capacity and isobaric thermal expansivity at temperatures between (253.15 and 473.15) $\mathrm{K}$ at pressures up to $400 \mathrm{MPa}$. The densities obtained in this way are in very close agreement with those reported by Lin and Trusler, but the isobaric specific heat capacity and the isobaric expansivity both differ significantly in the extremes of low temperatures and high pressures.
\end{abstract}

Keywords: equation of state; speed of sound; thermodynamic integration; water. 


\section{Introduction}

The determination of the thermodynamic properties of compressed liquids indirectly from the speed of sound has some advantages compared with direct measurements. These advantages arise in part because the speed of sound can be determined rapidly and automatically over wide ranges of temperature and pressure and with low uncertainty. Reliable measurements of sound speed can be carried out even under extreme pressures (e.g. up to $1 \mathrm{GPa}$ [1]), whereas direct density measurements are typically limited to maximum pressures on the order of $100 \mathrm{MPa}$. The formal method of determining other thermodynamic properties from measurements of the speed of sound is by means of thermodynamic integration. This approach, described in detail below, does not require assumptions to be made about the mathematical form of the equation of state and requires as inputs, in addition to the speed of sound, only initial values of density and heat capacity along one isobar [2]. A limitation of the method is that it cannot be applied to a single sound-speed datum; instead the analysis is carried out over a domain of temperature and pressure within which the speed of sound is known. This domain is usually bounded at low pressure by the isobar along which the initial values of density and heat capacity are specified.

Because the method of thermodynamic integration applies to a sound-speed surface, and not to a single datum, it is difficult to determine the uncertainty in the derived properties arising from uncertainties in the experimental speeds of sound. Furthermore, it is unduly onerous to measure the sound speed at every grid point used in the process of thermodynamic integration; instead either a surface fit or an interpolation method is employed and this may give rise to errors that exceed the experimental uncertainty. Finally, as already noted, the process requires initial values of density and isobaric heat capacity. These are usually specified by means of empirical functions fitted to experimental data and are inevitably burdened by a combination of experimental and fitting errors. Taking these observations together, it can be concluded that estimating the uncertainties of the derived properties is challenging.

In this work, we make specific reference to water, which is one of the most well studied of all substances. The thermodynamic properties of the fluid phases of water have been represented by a fundamental equation of state developed by Wagner and Pruss [3], which was adopted by the International Association for the Properties of Water and Steam (IAPWS) as the IAPWS Formulation 1995 for the Thermodynamic Properties of Ordinary Water Substance [4]. Recently, there has been renewed interest in the properties of water in a regime extending from the neighbourhood of the solid-liquid phase boundary into the metastable subcooled liquid region [5; 6; 7]. In 2012, new measurements of the speed of sound in compressed liquid water were published by Lin and Trusler [8]. These extend in temperature from $253.15 \mathrm{~K}$ to $473.15 \mathrm{~K}$ with pressures up to $400 \mathrm{MPa}$ and include data in the vicinity of the solid-liquid phase boundary. Lin and Trusler combined their new data with values of density and isobaric heat capacity obtained from the IAPWS formulation along an isobar at low pressure to obtain the thermodynamic properties of the compressed liquid. The rationale behind this approach was that the IAPWS formulation is more accurate at low pressures than at high pressures [3], so that initial values at low pressure $(0.1 \mathrm{MPa})$ combined with the new sound-speed data might lead to properties of liquid water with improved accuracy at high pressures. Densities obtained from the thermodynamic integration were found to agree with IAPWS-95 to within its 
uncertainty at all states; indeed, it was suggested on the basis of the results that the uncertainty associated with IAPWS-95 could be reduced. In the case of the isobaric heat capacity, the values derived from the speed of sound were also in good agreement with IAPWS-95, except at the lowest temperature considered. However, Holten et al. [6] have since suggested that the empirical correlation used by Lin and Trusler [8] to represent their very accurate speed of sound may not have been accurate enough to support fully the conclusions reached. Indeed, while the experimental data had relative uncertainties of between $0.03 \%$ and $0.04 \%$ at $95 \%$ confidence, the correlating equation only represented the data to within $\pm 0.15 \%$.

In this paper, we detail an improved methodology for estimating the uncertainties in thermodynamic properties determined from the speed of sound by thermodynamic integration, and we illustrate the propagation of errors by means of numerical simulations for liquid water based on the IAPWS-95 formulation. We then return to the experimental speeds of sound in pure water reported by Lin and Trusler [8] and describe a much more accurate surface fit. Finally, a revised thermodynamic integration has been carried out and the results are discussed. The behaviour of these new results in the region at $T<300 \mathrm{~K}$ has also been discussed in the recent paper of Wagner and Thol [7].

\section{Error Propagation in Thermodynamic Integration}

The method of thermodynamic integration permits the density $\rho$ and other thermodynamic properties of a fluid to be obtained from the speed of sound $c$ by integration of the following pair of partial differential equations within some specified domain of temperature $T$ and pressure $p$ [2]:

$$
\begin{gathered}
\left(\frac{\partial \rho}{\partial p}\right)_{T}=\frac{1}{c^{2}}+\frac{T \alpha^{2}}{c_{p}} \\
\left(\frac{\partial c_{p}}{\partial p}\right)_{T}=-T\left(\frac{\partial^{2} v}{\partial T^{2}}\right)_{p} .
\end{gathered}
$$

Here, $c_{p}$ is isobaric specific heat capacity, $\alpha=-\rho^{-1}(\partial \rho / \partial T)_{p}$ is isobaric expansivity, and $v=\rho^{-1}$ is specific volume, where $\rho$ is the mass density. The integration of these equations is subject to initial values on $\rho$ and $c_{p}$ that are usually specified along an isobar at $p=p_{0}$. The specification of $\rho\left(T, p_{0}\right)$ must support double differentiation with respect to temperature so that the right-hand side of equation (2) may be determined accurately. Various procedures may be used to integrate the differential equations numerically but, provided that due attention is paid to truncation and round-off errors, the results are not sensitive to the algorithm used. There is however a caveat to this statement in relation to the temperature derivatives of $\rho$, which must be evaluated at every grid point used in the integration. Numerical differentiation based on a small number of adjacent grid points can give erroneous derivatives and lead to a serious loss of accuracy. On the other hand, fitting a smoothing function along each isobar and differentiating that function may suppress oscillatory errors but there is a risk of biasing the solution with an inappropriate choice of smoothing function. 
In the following, it is assumed that the speed of sound surface to be used in the numerical integration is subject to a perturbation such that the perturbed sound speed $c^{\prime}$ is related to the true sound speed $c$ as follows:

$$
\frac{1}{c^{\prime 2}}=\frac{1+\varepsilon f_{1}}{c^{2}}
$$

where $\varepsilon$ is a dimensionless parameter and $f_{1}$ is a dimensionless function, generally depending upon both temperature and pressure. In the presence of this perturbation, all derived properties will also be perturbed. We assume here that $f_{1}$ is on the order of unity and that $\varepsilon \ll 1$ such that terms in the derived properties on the order of $\varepsilon^{2}$ and above are negligible. In that case, the density $\rho^{\prime}$ obtained by numerical integration will differ from the true density $\rho$ according to a first-order relation and may be written as

$$
\rho^{\prime}=\rho\left(1+\varepsilon f_{2}\right),
$$

where $f_{2}$ is a dimensionless function of temperature and pressure to be determined. Generally, $f_{2}(T, p)$ will be constrained by a function $f_{2,0}(T)=f_{2}\left(T, p=p_{0}\right)$ associated with errors in the initial values of $\rho$ specified along the isobar at $p=p_{0}$, and also be influenced at other pressures through the role of $f_{1}(T, p)$.

In the present analysis, the perturbations to be considered are such that $f_{1}$ and $f_{2}$ are both smooth, continuous and differentiable. That being the case, the isothermal pressure derivative of the perturbed density is

$$
\left(\frac{\partial \rho^{\prime}}{\partial p}\right)_{T}=\left(\frac{\partial \rho}{\partial p}\right)_{T}+\varepsilon\left[f_{2}\left(\frac{\partial \rho}{\partial p}\right)_{T}+\rho\left(\frac{\partial f_{2}}{\partial p}\right)_{T}\right]
$$

and the perturbed isobaric expansivity $\alpha^{\prime}$ is

$$
\alpha^{\prime}=\alpha-\varepsilon\left(\partial f_{2} / \partial T\right)_{p},
$$

correct to first order in $\varepsilon$. In the case of the perturbed isobaric specific heat capacity $c_{p}^{\prime}$, it is convenient to define an additional function $f_{3}$ such that

$$
c_{p}^{\prime}=c_{p}\left(1+\varepsilon f_{3}\right),
$$

again correct to first order in $\varepsilon$. This function will also be constrained at $p=p_{0}$ by a function $f_{3,0}(T)=f_{3}\left(T, p=p_{0}\right)$ associated with errors in the initial values of $c_{p}$ specified along that isobar.

To determine the relation between the three functions $f_{1}, f_{2}$ and $f_{3}$, the quantities $\rho, c, \alpha$ and $c_{p}$ in equation (1) are each replaced by the corresponding primed term, $\left(\alpha^{\prime 2} / c_{p}^{\prime}\right)$ is expanded as a power series in $\varepsilon$, and the first-order terms are collected with the following result:

$$
\rho\left(\frac{\partial f_{2}}{\partial p}\right)_{T}=\left(\frac{f_{1}-f_{2}}{c^{2}}\right)-\left(\frac{T \alpha^{2}}{c_{p}}\right)\left[f_{3}+f_{2}+\frac{2}{\alpha}\left(\frac{\partial f_{2}}{\partial T}\right)_{p}\right] .
$$

A second connection between $f_{2}$ and $f_{3}$ follows from equation (2):

$$
\left(\frac{\partial\left(c_{p} f_{3}\right)}{\partial p}\right)_{T}=T\left(\frac{\partial^{2}\left(f_{2} / \rho\right)}{\partial T^{2}}\right)_{p}
$$


correct to first-order in $\varepsilon$.

To study the propagation of errors associated with the method of thermodynamic integration, equations (8) and (9) may be solved simultaneously by numerical integration, subject to both an assumed perturbation function $f_{1}(T, p)$ and initial values $f_{2,0}(T)$ and $f_{3,0}(T)$. The simplest numerical procedure is a first-order Euler-type method as follows. The functions $f_{1}(T, p), f_{2,0}(T)$ and $f_{3,0}(T)$, together with the thermodynamic properties of the fluid, are used with equations (8) and (9) to obtain the values of $\left(\partial f_{2} / \partial p\right)_{T}$ and $\left[\partial\left(c_{p} f_{3}\right) / \partial p\right]_{T}$ along the initial isobar at $p=p_{0}$. The values of $f_{2}(T)$ and $f_{3}(T)$ along the isobar at $p_{1}=p_{0}+\delta p$, where $\delta p$ is a suitably chosen increment, are then obtained as follows:

$$
\begin{gathered}
f_{2}\left(T, p_{1}\right)=f_{2}\left(T, p_{0}\right)+\left(p_{1}-p_{0}\right)\left(\frac{\partial f_{2}}{\partial p}\right)_{T, p=p_{0}}, \\
f_{3}\left(T, p_{1}\right)=\left[\frac{c_{p}\left(T, p_{0}\right)}{c_{p}\left(T, p_{1}\right)}\right] f_{3}\left(T, p_{0}\right)+\left[\frac{\left(p_{1}-p_{0}\right)}{c_{p}\left(T, p_{1}\right)}\right]\left(\frac{\partial\left(c_{p} f_{3}\right)}{\partial p}\right)_{T, p=p_{0}} .
\end{gathered}
$$

Pressure $p_{1}$ then becomes the initial pressure and the procedure is repeated until the maximum pressure of interest is reached. This procedure requires estimates of the thermodynamic properties of the fluid and, in the present case, those properties were obtained from the equation of state of Wagner and Pruss [3]. An alternative numerical approach is to apply the conventional numerical integration procedure with equations (1) and (2) with and without imposed perturbations to the sound speed surface and initial values.

\section{Error Propagation Scenarios}

To illustrate the propagation of errors for the case of water, we consider several scenarios relating to the functions $f_{1}(T, p), f_{2,0}(T)$ and $f_{3,0}(T)$. Since we are considering small perturbations, it is sufficient to consider plausible scenarios for these perturbations separately. For the purposes of this discussion, we consider imposed perturbation functions defined as follows:

$$
\begin{gathered}
f_{1}(T, p)=A_{1} \cos \left(k_{1} T\right) \cos \left(k_{4} p\right), \\
f_{2,0}(T)=A_{2} \cos \left(k_{2} T\right), \\
f_{3,0}(T)=A_{3} \cos \left(k_{3} T\right),
\end{gathered}
$$

where the amplitudes $A_{1}, A_{2}$ and $A_{3}$ are either unity or zero, depending upon whether the perturbation is switched on or off, and $k_{i} \geq 0(i=1,4)$. This allows both constant and variable perturbations that can be combined to represent smoothly-varying errors in the sound speed and the initial values. The simple first-order method described above was implemented with a pressure increment of $\delta p=1 \mathrm{MPa}$. The temperature derivatives that appear in equations (8) and (9) were calculated using five-point Lagrangian polynomials centred on the temperature of interest and with a $5 \mathrm{~K}$ spacing between nodes. The results were not sensitive to the value of the pressure increment or to increasing the temperature increment to $10 \mathrm{~K}$. However, smaller temperature increments did give rise to numerical instability as evidenced by the appearance of additional oscillations in $f_{2}$ and $f_{3}$. 
The cases in which $k_{i}=0(i=1,4)$ are illustrated in Figure 1. Figures 1 (a) and (b) show the density and heat-capacity perturbation functions arising when $f_{1}=1$ and $f_{2,0}=f_{3,0}=0$. In this case, the sound-speed perturbation gives rise to a nearly constant error in the isothermal compressibility and the resulting density perturbation $f_{2}$ increases with increasing pressure and is only weakly dependent upon temperature. The relative density perturbation is roughly one order of magnitude smaller than the sound speed perturbation in this scenario. The corresponding heat-capacity perturbation $f_{3}$ also increases in magnitude with increasing pressure but has a more complex dependence upon temperature, with rapid variations at the lower-end of the temperature range. Figures 1 (c) and (d) show $f_{2}$ and $f_{3}$ for the case in which $f_{2,0}=1$ and $f_{1}=f_{3,0}=0$. In this case, $f_{2}$ is of course unity at the initial pressure and it declines slightly with increasing pressure, consistent with the term $-f_{2} / c^{2}$ in equation (8), with little dependence upon temperature. The heat-capacity perturbation is constrained to zero at the initial pressure and increases in magnitude with pressure, developing a distinct temperature dependence. Figures 1 (e) and (f) show the results when $f_{3,0}=1$ and $f_{1}=f_{2,0}=0$. In this case, the density perturbation function $f_{2}$ is initially zero but rapidly increases in magnitude with an increase in pressure. The strong dependence upon temperature reflects mainly the temperature dependence of $\alpha$. The heat-capacity perturbation increases with increasing pressure at a rate that is strongly dependent upon temperature, this time reflecting mainly the temperature derivative of $\alpha$. A notable feature of all the scenarios considered in Figure 1 is that $\left|f_{2}\right|$ and $\left|f_{3}\right|$ are on the order of unity or smaller, so that small constant relative errors in $c^{2}$ or in the initial values $f_{2,0}$ and $f_{3,0}$ give rise to correspondingly small errors in the derived properties. This accords with the analysis reported by Lin and Trusler [8].

When the perturbation arises solely from the sound speed surface, $A_{1}=1, A_{2}=A_{3}=0$, and both $f_{2}$ and $f_{3}$ are related to the generating function $f_{1}$ alone. Having considered in Figure 1 the case when $k_{1}=0$, we explore in Figures 2 to 4 further scenarios in which $k_{1}=(0.05,0.1$ or $0.2) \mathrm{K}^{-1}$ with $k_{4}=(0$ or 0.02$) \mathrm{MPa}^{-1}$. These correspond to periodic $f_{1}$ perturbation functions with temperature wavelengths of between (31 and 125) $\mathrm{K}$ and pressure wavelengths of either $314 \mathrm{MPa}$ or infinity. Figure 2 shows the relative density perturbation function $f_{2}$ in these scenarios with parts (a) to (c) corresponding to $k_{4}=0$ and parts (d) to (f) corresponding to $k_{4}=0.02 \mathrm{MPa}^{-1}$. It can be seen that, with increasing pressure, $f_{2}$ rapidly develops an oscillating dependence upon $T$ that reflects that of $f_{1}$. The effect of the sound-speed perturbation is reduced when $k_{4}>0$ as the influence of $f_{1}$ at a given $T$ oscillates with increasing pressure rather than being constant. The most important conclusion is that $f_{2}$ remains in magnitude about one order smaller than $f_{1}$, so that small errors in the sound speed surface, whatever their dependence upon temperature or pressure, give rise to smaller errors in the derived densities, especially when they oscillate in sign with increasing pressure.

Figure 3 shows the heat-capacity perturbation function for the same scenarios as illustrated in Figure 2. Like $f_{2}, f_{3}$ rapidly develops an oscillating dependence upon $T$ that reflects that of the sound-speed perturbation function. However, the amplitude of these oscillations increases rapidly with $k_{2}$. Together Figure 1 (b) and Figures 4 (a) to (c) cover the cases in which $k_{1}=(0.00,0.05,0.1$ and 0.2$)$ and the amplitude of $f_{3}$ is seen to increase over that range by about two orders of magnitude. Again, the effect of the sound-speed perturbation function is reduced when $k_{4}>0$. Since the thermal expansivity $\alpha$ is an important derived property, we illustrate the same scenarios for that property in Figure 4. The quantity plotted here is 
$-T\left(\partial f_{2} / \partial T\right)_{p}$, which is the coefficient of $\varepsilon$ in the dimensionless property $T \alpha^{\prime}$ and thus indicative of the absolute, rather than relative, error in the thermal expansivity. Although not plotted in Figure 1 , when $f_{1}=1,\left|T\left(\partial f_{2} / \partial T\right)_{p}\right|$ is on the order of $10^{-1}$. Thus the overall behaviour of $-T\left(\partial f_{2} / \partial T\right)_{p}$ is somewhat similar to that of $f_{3}$ but with less-rapid growth in amplitude as $k_{1}$ is increased. When $k_{4}>0$, the influence of the sound-speed perturbation function is reduced but less strongly than was the case for $f_{3}$. The overall conclusion from Figures 2 and 3 is that short-wavelength temperature fluctuations are the most toxic components of $f_{1}$ from the perspective of the derived heat capacity and thermal expansivity.

To complete the picture, we illustrate in Figures 5 and 6 the effects of errors in the initial values under the assumption of an error-free sound-speed surface. Figure 5 addresses the cases in which $f_{1}=f_{3,0}=0$ with $f_{2,0}$ given by equation (13) and $k_{2}=(0.05$ or 0.2$)$. As shown in Figures 5 (a) and (d), the initial error in density maintains roughly the same dependence upon temperature and does not grow in amplitude with increasing pressure. Figures 5 (b) and (e) show that the heat-capacity perturbation function initially grows rapidly in amplitude with increasing pressure but the rate of growth slows at higher pressures. Figures 5 (c) and (f) show that the amplitude of $-T\left(\partial f_{2} / \partial T\right)_{p}$ declines slightly as the pressure increases. Both $f_{3}$ and $-T\left(\partial f_{2} / \partial T\right)_{p}$ are much larger than the perturbation $f_{2,0}$ from which they arise and, again, the most toxic perturbations are those with the shortest wavelength in temperature. Figure 6 addresses the case in which $f_{1}=f_{2,0}=0$ with $f_{3,0}$ given by equation (14) and $k_{2}=(0.05$ or 0.2$)$. In this case, the arising density perturbation function $f_{2}$ remains very small, while $f_{3}$ maintains an almost constant amplitude with increasing pressure. The expansivity perturbation term $-T\left(\partial f_{2} / \partial T\right)_{p}$ also maintains a small amplitude.

To summarise this section, we find that perturbations in either the sound-speed surface or the initial density function $\rho\left(T, p_{0}\right)$ exhibiting short-wavelength temperature fluctuations give rise to much larger errors in the derived properties than found for constant or slowly-varying errors. Thus it is important that mathematical functions used to represent experimental sound-speed data for the purposes of thermodynamic integration are constructed such that deviations are slowly-varying functions of temperature, especially free from oscillations in temperature that persist over the pressure range.

\section{Application to Water}

The function used by Lin and Trusler [8] to represent their sound-speed data was not able to do so within the experimental uncertainty. This fact is illustrated in Figure 7 where the deviations of the experimental data from the correlation of Lin and Trusler are plotted as a function of temperature along isobars. The deviations substantially exceed the reported experimental relative uncertainties of (0.03 to 0.04$) \%$ at $95 \%$ confidence. There is also evidence of oscillatory behaviour that, as discussed above, might give rise to larger errors in the isobaric heat capacity and isobaric expansivity. In order to reduce these errors, a new correlation of the same sound-speed data for water has been developed in this work and used in a new thermodynamic integration to obtain improved derived properties of compressed liquid water. 
The new correlation was developed in two stages, one for the speed of sound at $p=0.1 \mathrm{MPa}$, and one for the additional contribution (or residual function) at higher pressures. Because of the large change in pressure with a small change in speed of sound at constant temperature, it was necessary to develop a pressure explicit equation with independent variables of temperature and speed of sound for the residual function.

In the integration over pressure of equations (1) and (2) to obtain densities, heat capacities, etc., a low pressure limit is required. This limit is arbitrary, and could be a pressure of zero, atmospheric, or any other value. For this work, the lower bound was set to $0.1 \mathrm{MPa}$ for the following reasons:

- A pressure of zero cannot be reached for liquid states.

- The lower pressure limit in the liquid varies with temperature (i.e., the vapor pressure curve).

- The use of a moderate or high pressure for the lower limit requires switching the integration bounds for calculations at low pressures.

- The difference in speed of sound between the vapor pressure state and $0.1 \mathrm{MPa}$ is mostly insignificant below the boiling point (with the largest difference being $0.011 \%$ at the triple point).

The equation of state from Wagner and Pruss [3] is a high-accuracy fit that uses the Helmholtz energy as the fitted property with independent variables of temperature and density. The Helmholtz energy is one of four fundamental properties from which all other thermodynamic properties can be calculated through simple derivatives. For example, the pressure is the first derivative of the Helmholtz energy with respect to density, and the isochoric heat capacity is the second derivative of the Helmholtz energy with respect to temperature. The use of this fundamental characteristic enables all measurements for different properties to be combined in a single fit to obtain the Helmholtz energy equation, thus resulting in a correlation with uncertainties lower than those of the measurements (for fluids that have been well measured with multiple data types). Thus, calculations from the Wagner-Pruss equation at $0.1 \mathrm{MPa}$ were used rather than measurements for the following reasons:

- The Wagner-Pruss equation was developed with the lowest uncertainties possible through the use of a combination of all data types.

- Data measured at near atmospheric pressures are not located exactly at $0.1 \mathrm{MPa}$, so a correction would be required (although this would generally be very small).

- A significant portion of the high-accuracy measurements of the speed of sound do not pertain to atmospheric pressure (except in the metastable region).

- The most accurate data currently available, those of Lin and Trusler, have a lower pressure limit of $0.98 \mathrm{MPa}$.

With the use of the Wagner-Pruss [3] equation of state, values were calculated at temperatures between (234 and 600$) \mathrm{K}$ at a pressure of $0.1 \mathrm{MPa}$. Calculated values above $372.76 \mathrm{~K}$ are metastable liquid states and values below $273.15 \mathrm{~K}$ represent supercooled liquid states, both of which were extrapolated from the equation of state either within the two-phase region or below the melting line of water. Of these data, values at temperatures between (256 and 492) $\mathrm{K}$ were fitted. The low and high temperature data were calculated only as a check 
on the extrapolation behaviour of the equation [given further below in equations (13) and (14)]. Between (256 and 488) K, nearly all of the calculated points from the Wagner-Pruss equation of state were fitted to within $0.005 \%$ (50 ppm). Deviations are less than $0.1 \%$ between (250 and 256) $\mathrm{K}$ and between (488 and 510) $\mathrm{K}$. At low temperatures, the deviation reaches $2.8 \%$ at $236 \mathrm{~K}$. The deviations increase substantially above $510 \mathrm{~K}$ due to the rising change in properties approaching the critical point.

The contribution (or residual function) for high pressures was developed by first subtracting the value of the speed of sound at $p=0.1 \mathrm{MPa}$ from each measured point of Lin and Trusler [8]. The fitting algorithm of Lemmon and Jacobsen [9] was modified to directly fit pressure (as a function of temperature and speed of sound) instead of the Helmholtz energy. This algorithm has been developed over the last two decades for the development of equations of state. The non-linear fitting algorithm simultaneously fits all parameters of an equation, including both coefficients and exponents. This results in equations with about half as many terms as those obtained linearly, and with less intercorrelation between the terms, resulting in better extrapolation abilities. Furthermore, constraints can be placed on the fit to control any aspect of the equation, such as the extrapolation to high temperatures or pressures, or limits can be placed on the values of the parameters. For example, a constraint can specify that the slope of an isobar should always be positive and that the curvature should always be negative. These features were applied to help shape the final functional form developed here.

The independent variables in the residual function for pressure are temperature and speed of sound. During the fitting process, the number of terms was kept to the absolute minimum to reduce oscillations, rather than including extra terms that could help obtain lower uncertainties. The deviations were monitored and points with slightly high or low values were removed from the fit to maintain a very smooth surface with no irregularities introduced from the points with the largest uncertainties. Because the residual function goes to zero at $0.1 \mathrm{MPa}$, deviations appear larger for low pressures and it was important to not overfit these states. For example, the deviation for the residual pressure for the measured point at $373.18 \mathrm{~K}$ and $1.07 \mathrm{MPa}$ is $15 \%$, but the deviation in the total speed of sound is $0.0015 \%$. Of the 206 data points from Lin and Trusler at pressures greater than $2 \mathrm{MPa}$, the final fit used 166 points. The deviations for about $85 \%$ of the 206 points are less than $0.2 \%$ in pressure. This translates to an upper limit of about $0.02 \%$ in the speed of sound for pressures below $200 \mathrm{MPa}$ as shown in Figure 8. A comparison with Figure 7 shows that the amplitude of the deviations has been reduced by about a factor of 5 from those obtained in the work of Lin and Trusler.

The final equation obtained is

$$
p / p_{0}=0.1+c_{4} T^{t_{4}} \eta^{d_{4}}+\sum_{i=5}^{7} c_{i} T^{t_{i}} \eta^{d_{i}} \exp \left(-a_{i} \eta^{\varphi_{i}}\right)+\sum_{i=8}^{9} c_{i} T^{t_{i}} \eta^{d_{i}} \exp \left(-a_{i} \eta^{\varphi_{i}}-b_{i} T^{Y_{i}}\right),
$$

where $p_{0}=1 \mathrm{MPa}, \tau=(600 \mathrm{~K}) / T$ and $\eta=\left(c-c_{0}\right) /\left(700 \mathrm{~m} \cdot \mathrm{s}^{-1}\right)$. Here, $c_{0}$ is the speed of sound at $p=0.1 \mathrm{MPa}$, which is represented by the following equation:

$$
c_{0} /\left(\mathrm{m} \cdot \mathrm{s}^{-1}\right)=\sum_{i=1}^{3} c_{i}(\tau / 2)^{t_{i}}
$$


The coefficients of equations (15) and (16) are given in Table 1. The reducing values of $600 \mathrm{~K}$ and $700 \mathrm{~m} \cdot \mathrm{s}^{-1}$ were obtained simultaneously with the other parameters in Table 1 during the fitting process [the three terms (six fitted parameters) used in equation (16) were fitted separately]. These reducing values were the best possible in order for this functional form to represent the speed of sound measurements with the lowest uncertainty.

Table 1. Coefficients in equations (15) and (16)

\begin{tabular}{cccccccc}
\hline$i$ & \multicolumn{1}{c}{$c_{i}$} & \multicolumn{1}{c}{$d_{i}$} & $a_{i}$ & \multicolumn{1}{c}{$\varphi_{i}$} & $b_{i}$ & $\gamma_{i}$ \\
\hline 1 & 2272.27 & 0.5 & & & & & \\
2 & -734.963 & 2.8 & & & & & \\
3 & -35.7119 & -4.5 & & & & & \\
4 & 445.376 & 2.15 & 0.579 & & & & \\
5 & -337.85 & 4.647 & 0.59 & 0.65 & 0.83 & & \\
6 & -64.5124 & 6.7 & 0.60643 & 1.02 & 0.972 & & \\
7 & 207.293 & 6.0 & 0.6 & 0.91 & 0.9272 & & \\
8 & -1557.72 & 1.59 & 0.7 & 0.07 & 1.64 & 0.08 & 5.233 \\
9 & 1462.51 & 2.0 & 0.7 & 0.12 & -0.183 & 0.1 & 5.0 \\
\hline
\end{tabular}

Derived thermodynamic properties of compressed liquid water were obtained by numerical integration of equations (1) and (2) with the use of the same initial values and integration algorithm as described by Lin and Trusler [8], but with the new sound-speed correlation. The integration domain extends in temperature from $253.15 \mathrm{~K}$ to $473.15 \mathrm{~K}$ and in pressure from $0.1 \mathrm{MPa}$ to $400 \mathrm{MPa}$. Liquid states at $p=0.1 \mathrm{MPa}$ are metastable for temperatures below $273.15 \mathrm{~K}$ and above $372.76 \mathrm{~K}$. The results of the calculations are tabulated in the supplementary data and have been compared in detail with other experimental data and with the equations of state of Wagner-Pruss [3] and Holten et al. [6] in the paper of Wagner and Thol [7]. Accordingly, we restrict the discussion here to the differences in the derived properties associated with replacing the sound-speed correlation of Lin and Trusler with the present more-accurate formulation. The differences are found to be very small for all properties except near to the lower temperature limit. Somewhat arbitrarily, we divide the temperature range at $303.15 \mathrm{~K}$. For $T \geq 303.15 \mathrm{~K}$ and each property, we quantify these differences in terms of the average absolute relative difference $\Delta_{\mathrm{AAD}}$ [where the deviations are calculated as $\left(x_{\exp }-x_{\mathrm{fit}}\right) / x_{\mathrm{fit}}$, with $x$ as any property] and the maximum absolute relative difference $\Delta_{\text {MAD }}$. In the case of density, we have $\Delta_{\text {AAD }}=0.003 \%$ and $\Delta_{\text {MAD }}=0.012 \%$; for isobaric expansivity, we have $\Delta_{\mathrm{AAD}}=0.20 \%$ and $\Delta_{\mathrm{MAD}}=1.12 \%$; and for isobaric specific heat capacity we have $\Delta_{\mathrm{AAD}}=0.06 \%$ and $\Delta_{\mathrm{MAD}}=0.29 \%$. The average absolute relative differences are all much smaller than the corresponding uncertainties estimated by Lin and Trusler and we conclude that, in this temperature range, while the new results are to be preferred, the differences are mostly insignificant. The differences at $T \leq 303.15 \mathrm{~K}$ are illustrated in Figures 9, 10 and 11. Figure 9 shows that the relative difference of density becomes largest in the approach to the highest pressure and the lowest temperature. Figure 10 compares the present values of the isobaric expansivities with those obtained by Lin and Trusler. Unsurprisingly, the differences also increase in the approach to the highest pressure and the lowest temperature. Figure 11 shows a similar pattern for the isobaric specific heat capacity. For all three properties, the differences towards the extreme values of low $T$ and high $p$ exceed the 
uncertainties estimated by Lin and Trusler. This is attributed to the oscillatory nature of the sound-speed deviations as a function of temperature.

Another factor that might be important at low temperatures is that the thermodynamic integration path passes through metastable regions in which the speed of sound was not measured. This is a consequence of the negative slope of the melting pressure curve at $p \leq 209 \mathrm{MPa}$. In this region, we depend upon the correlating equation to provide an accurate estimation of the sound speed in the metastable regions. Although we cannot validate this estimation directly, we remark that our derived values of $\alpha$ were found to compare very favourably with the experimental data of Ter Minassian et al. [10] and also the recent equation of state of Holten et al. [6] in both the stable and metastable regions down to $T=253.15 \mathrm{~K}$.

\section{Conclusions}

This study highlights the fact that, in thermodynamic integration, errors in the speed of sound that vary rapidly with temperature give rise to much larger errors in the derived properties than do more slowly-varying errors. We present an analysis from which the consequences of any assumed perturbation to the sound-speed surface and/or the initial values of $\rho$ and $c_{p}$ can be computed, and we illustrate this with relevant scenarios for pure water. Finally, we report an improved correlation for the speed of sound in pure water, based on the experimental data of Lin and Trusler [8], and we report derived properties of water obtained from this surface by thermodynamic integration.

\section{Acknowledgements}

The authors are grateful to $\mathrm{V}$. Holten for sharing unpublished calculations of the effects of sinusoidal perturbation in the speed of sound on derived properties. We also acknowledge useful discussions with $\mathrm{W}$. Wagner, M. Thol and J. Sengers. 


\section{References}

[1] P.J. Kortbeek, M.J.P. Muringer, N.J. Trappeniers, S.N. Biswas, Rev. Sci. Instrum. 56 (1985) 1269-1273.

[2] J.P.M. Trusler, Physical Acoustics and Metrology of Fluids, Bristol, 1991.

[3] W. Wagner, A. Pruss, J. Phys. Chem. Ref. Data 31 (2002) 387-535.

[4] IAPWS, Revised Release on the IAPWS Formulation 1995 for the Thermodynamic Properties of OrdinaryWater Substance for General and Scientific Use, 2014., http://www.iapws.org/relguide/IAPWS-95.html (2014).

[5] V. Holten, C.E. Bertrand, M.A. Anisimov, J.V. Sengers, J. Chem. Phys. 136 (2012) 094507.

[6] V. Holten, J.V. Sengers, M.A. Anisimov, J. Phys. Chem. Ref. Data 43 (2014) 043101.

[7] W. Wagner, M. Thol, J. Phys. Chem. Ref. Data 44 (2015) 043102.

[8] C.W. Lin, J.P.M. Trusler, J. Chem. Phys. 136 (2012) 094511.

[9] E.W. Lemmon, R.T. Jacobsen, J. Phys. Chem. Ref. Data 34 (2005) 69-108.

[10] L.T. Minassian, P. Pruzan, A. Soulard, J. Chem. Phys. 75 (1981) 3064-3072. 
Figures
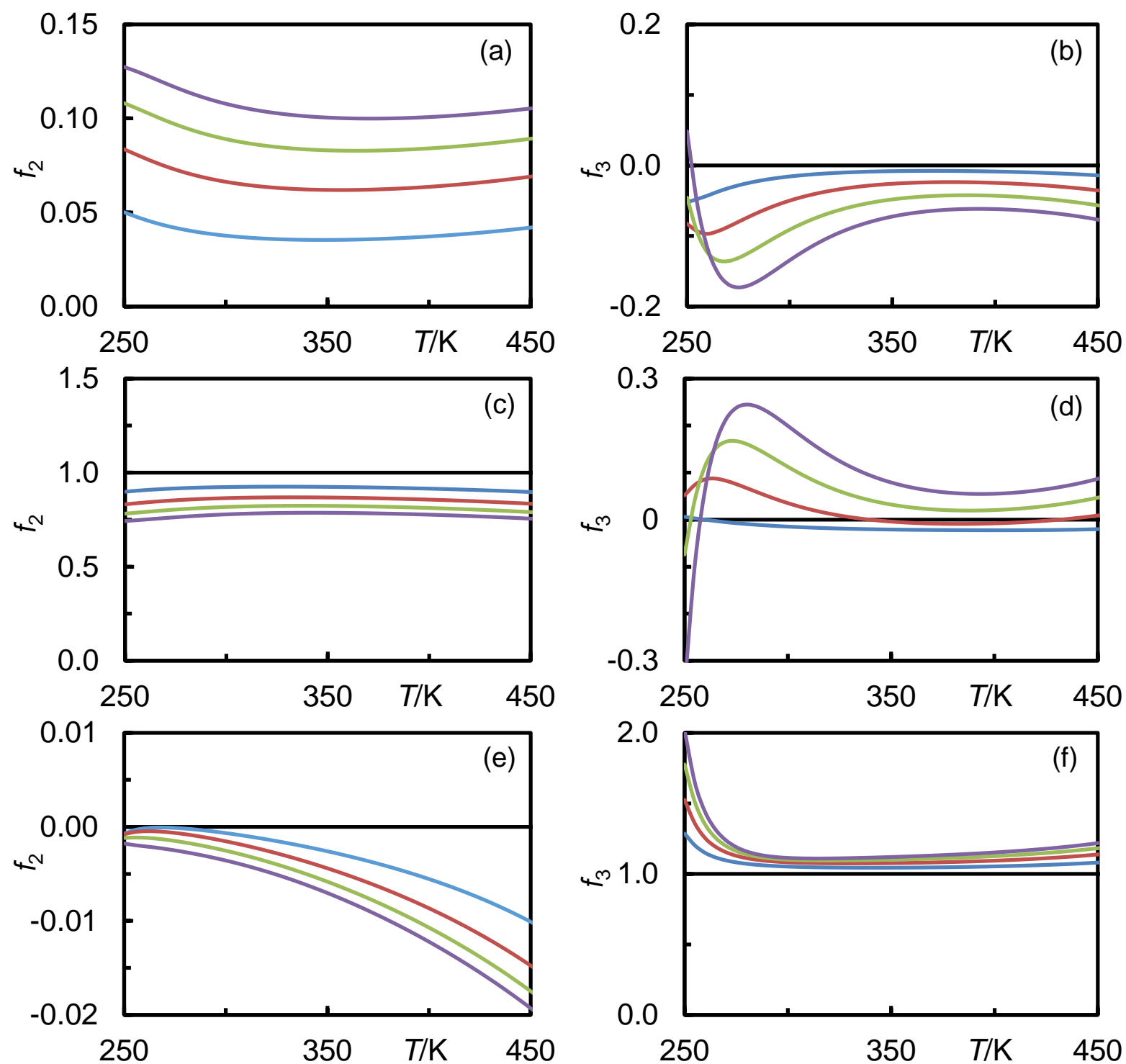

Figure 1. Density perturbation function $f_{2}$ and heat-capacity perturbation function $f_{3}$ as functions of temperature $T$ at various pressures arising from: (a) and (b), constant soundspeed perturbation $f_{1} ;(c)$ and (d), constant initial-density perturbation $f_{2,0} ;(e)$ and (f) constant initial heat-capacity perturbation $f_{3,0}$. Curves: black, $p=1 \mathrm{MPa}$; blue, $p=100 \mathrm{MPa}$; red, $p=200 \mathrm{MPa}$; green, $p=300 \mathrm{MPa}$; purple, $p=400 \mathrm{MPa}$. 

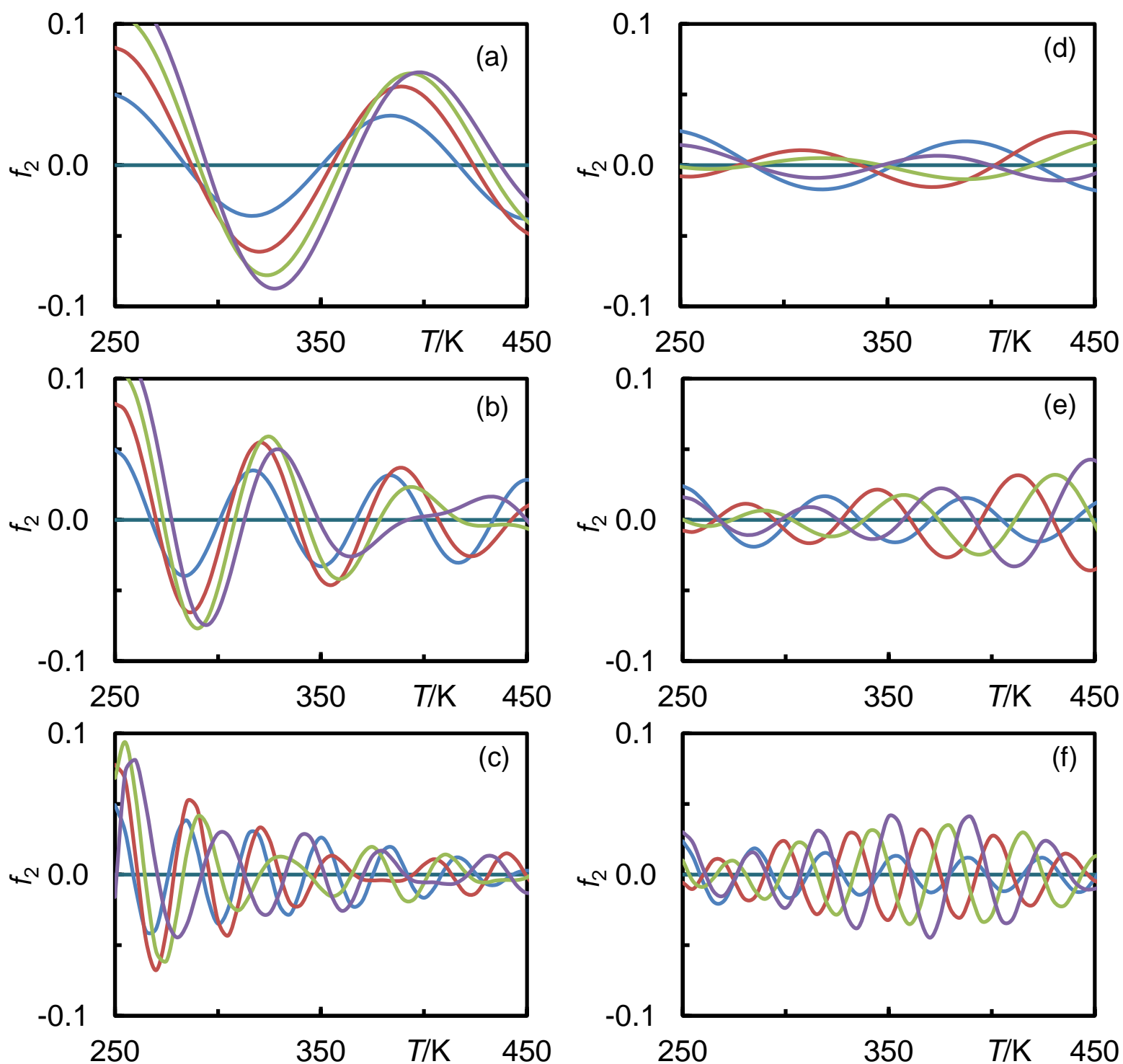

Figure 2. Density perturbation function $f_{2}$ as a function of temperature $T$ at various pressures $p$ arising from sound-speed perturbation $f_{1}$ as defined by equation (10) with $A_{1}=1$ and parameters as follows: (a) $k_{1}=0.05 \mathrm{~K}^{-1}, k_{4}=0 \mathrm{MPa}^{-1}$; (b) $k_{1}=0.1 \mathrm{~K}^{-1}, k_{4}=0 \mathrm{MPa}^{-1}$; (c) $k_{1}=0.2 \mathrm{~K}^{-1}, k_{4}=0 \mathrm{MPa}^{-1}$; (d) $k_{1}=0.05 \mathrm{~K}^{-1}, k_{4}=0.02 \mathrm{MPa}^{-1}$; (e) $k_{1}=0.1 \mathrm{~K}^{-1}, k_{4}=0.02 \mathrm{MPa}^{-1}$; (f) $k_{1}=0.2 \mathrm{~K}^{-1}, k_{4}=0.02 \mathrm{MPa}^{-1}$. Curves: black, $p=1 \mathrm{MPa}$; blue, $p=100 \mathrm{MPa}$; red, $p=200 \mathrm{MPa}$; green, $p=300 \mathrm{MPa}$; purple, $p=400 \mathrm{MPa}$. 

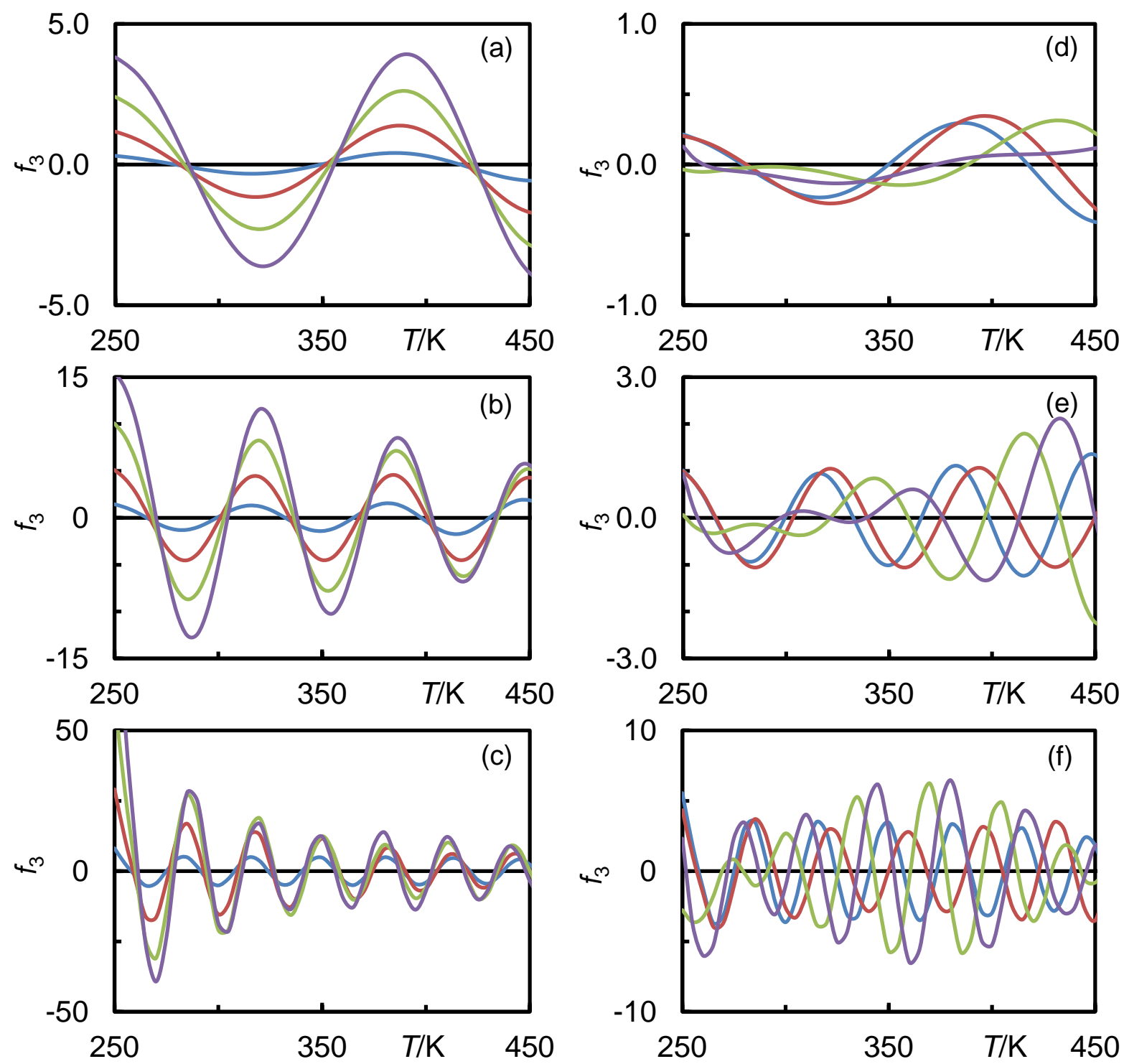

Figure 3. Heat-capacity perturbation function $f_{3}$ as a function of temperature $T$ at various pressures $p$ arising from sound-speed perturbation $f_{1}$ as defined by equation (10) with $A_{1}=1$ and parameters as follows: (a) $k_{1}=0.05 \mathrm{~K}^{-1}, k_{4}=0 \mathrm{MPa}^{-1}$; (b) $k_{1}=0.1 \mathrm{~K}^{-1}, k_{4}=0 \mathrm{MPa}^{-1}$; (c) $k_{1}=0.2 \mathrm{~K}^{-1}, k_{4}=0 \mathrm{MPa}^{-1}$; (d) $k_{1}=0.05 \mathrm{~K}^{-1}, k_{4}=0.02 \mathrm{MPa}^{-1}$; (e) $k_{1}=0.1 \mathrm{~K}^{-1}, k_{4}=0.02 \mathrm{MPa}^{-1}$; (f) $k_{1}=0.2 \mathrm{~K}^{-1}, k_{4}=0.02 \mathrm{MPa}^{-1}$. Curves: black, $p=1 \mathrm{MPa}$; blue, $p=100 \mathrm{MPa}$; red, $p=200 \mathrm{MPa}$; green, $p=300 \mathrm{MPa}$; purple, $p=400 \mathrm{MPa}$. 

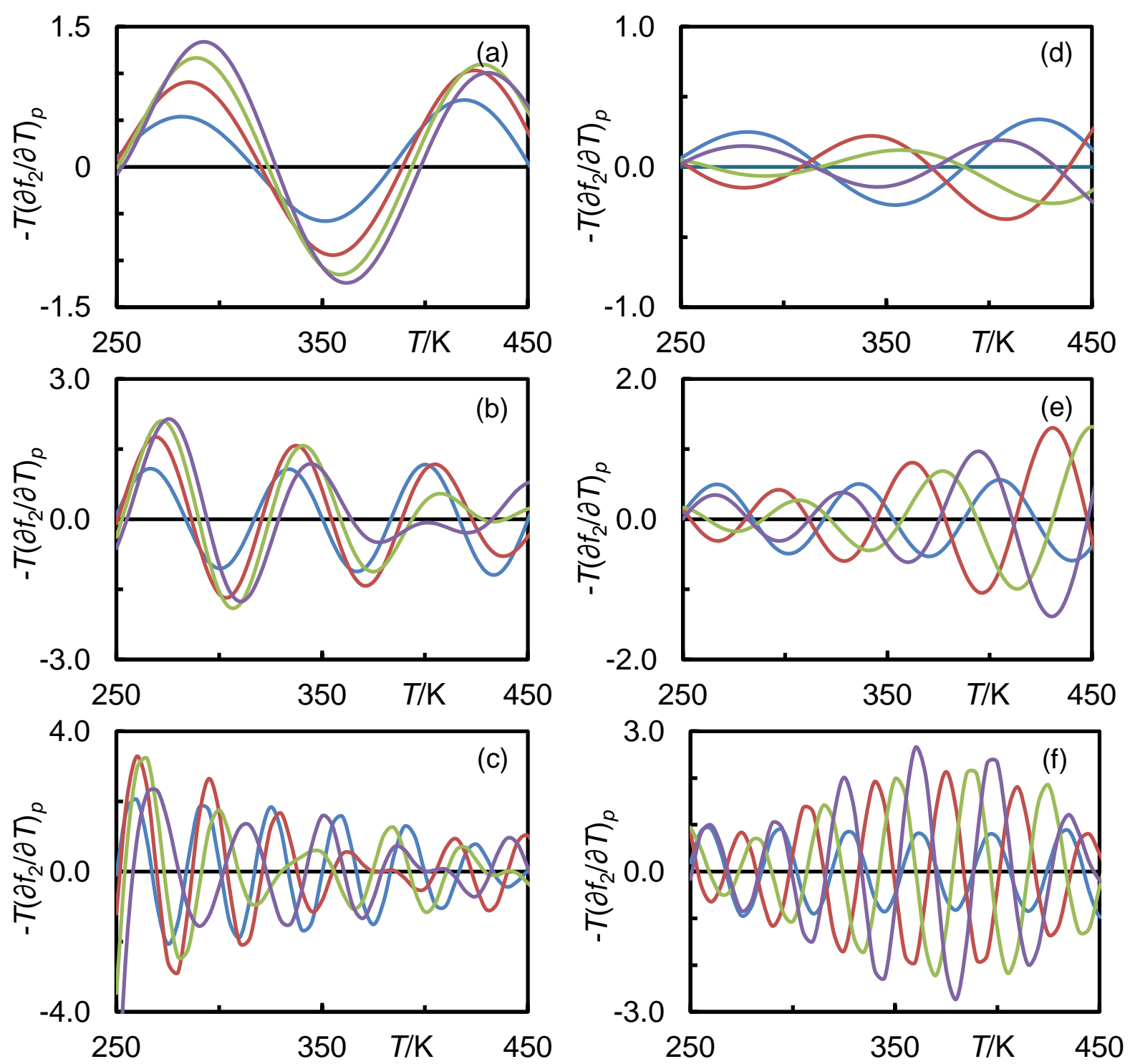

Figure 4. Isobaric-expansivity perturbation function $-T\left(\partial f_{2} / \partial T\right)_{p}$ as a function of temperature $T$ at various pressures $p$ arising from sound-speed perturbation $f_{1}$ as defined by equation (10) with $A_{1}=1$ and parameters as follows: (a) $k_{1}=0.05 \mathrm{~K}^{-1}, k_{4}=0 \mathrm{MPa}^{-1}$; (b) $k_{1}=0.1 \mathrm{~K}^{-1}$, $k_{4}=0 \mathrm{MPa}^{-1}$; (c) $k_{1}=0.2 \mathrm{~K}^{-1}, k_{4}=0 \mathrm{MPa}^{-1}$; (d) $k_{1}=0.05 \mathrm{~K}^{-1}, k_{4}=0.02 \mathrm{MPa}^{-1}$; (e) $k_{1}=0.1 \mathrm{~K}^{-1}$, $k_{4}=0.02 \mathrm{MPa}^{-1}$; (f) $k_{1}=0.2 \mathrm{~K}^{-1}, k_{4}=0.02 \mathrm{MPa}^{-1}$. Curves: black, $p=1 \mathrm{MPa}$; blue, $p=100 \mathrm{MPa}$; red, $p=200 \mathrm{MPa}$; green, $p=300 \mathrm{MPa}$; purple, $p=400 \mathrm{MPa}$. 

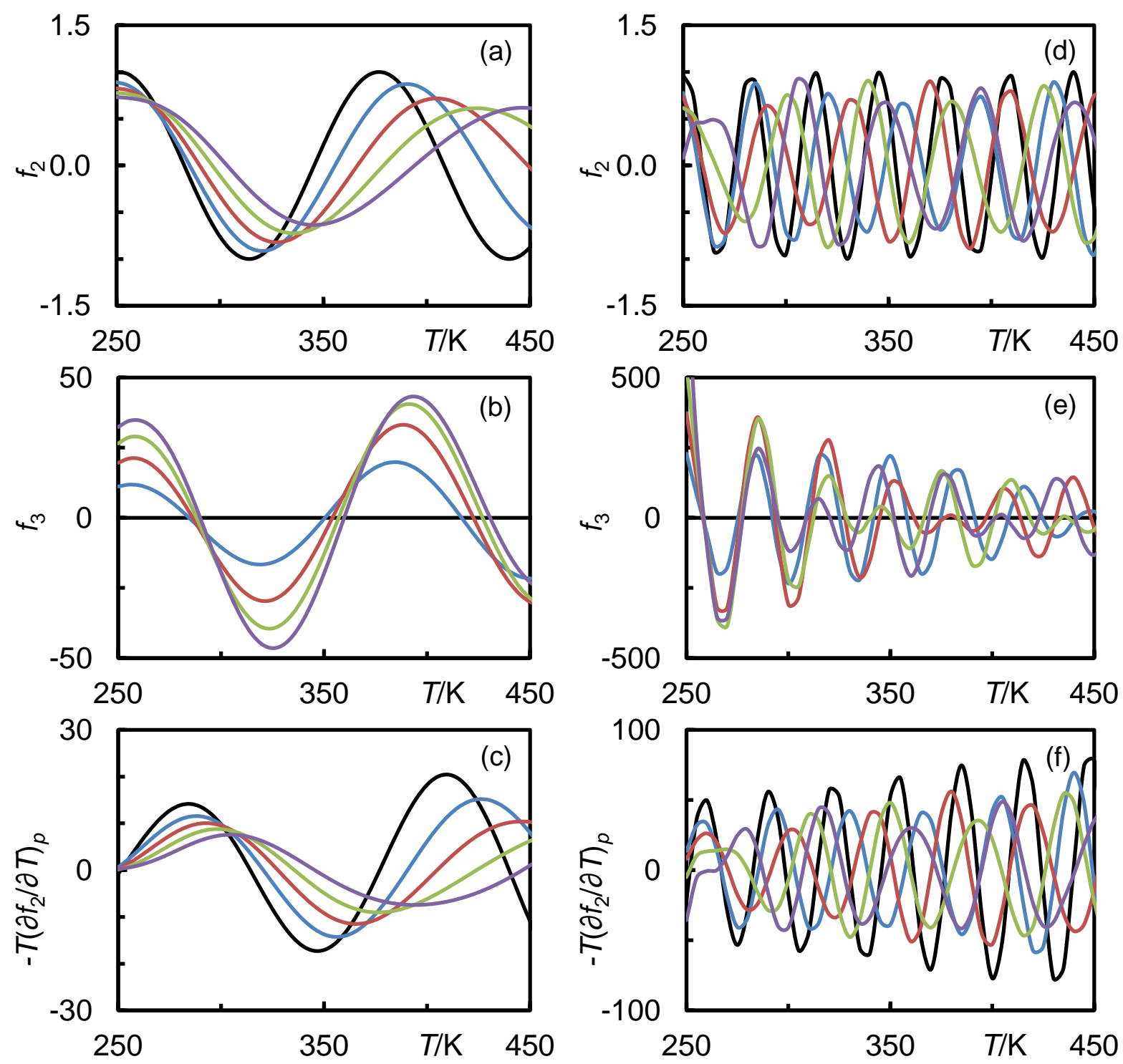

Figure 5. Effect of the initial-value density perturbation function $f_{2,0}(T)$ defined by equation (11) with $A_{2}=1$ on the density perturbation function $f_{2}$, heat-capacity perturbation function $f_{3}$ and isobaric-expansivity perturbation function $-T\left(\partial f_{2} / \partial T\right)_{p}$ as functions of temperature $T$ at various pressures $p$ : (a) to (c) $k_{1}=0.05 \mathrm{~K}^{-1}$; (d) to (f) $k_{1}=0.2 \mathrm{~K}^{-1}$. Curves: black, $p=1 \mathrm{MPa}$; blue, $p=$ $100 \mathrm{MPa}$; red, $p=200 \mathrm{MPa}$; green, $p=300 \mathrm{MPa}$; purple, $p=400 \mathrm{MPa}$. 

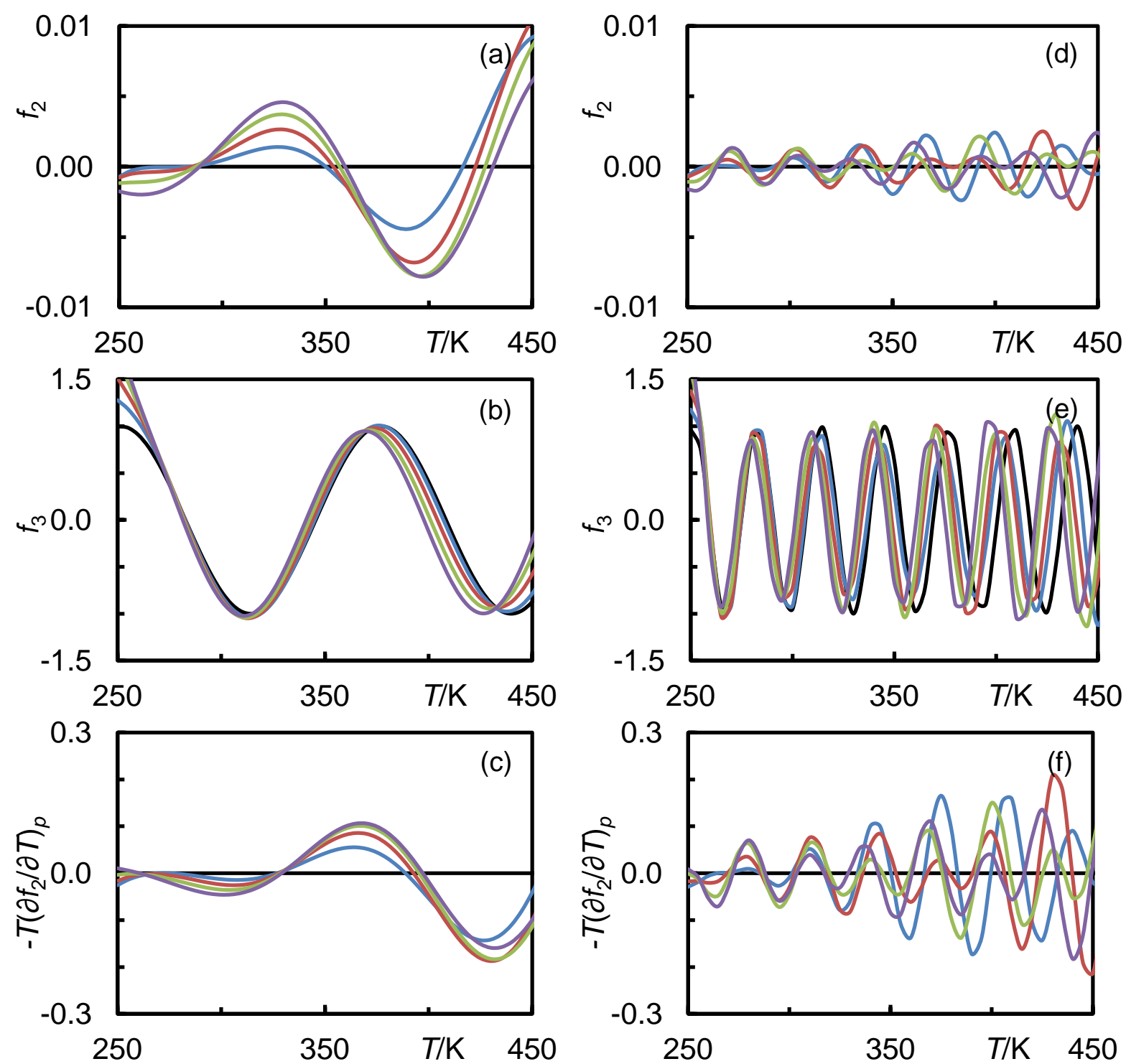

Figure 6. Effect of the initial-value heat-capacity perturbation function $f_{3,0}(T)$ defined by equation (12) with $A_{3}=1$ on the density perturbation function $f_{2}$, heat-capacity perturbation function $f_{3}$ and isobaric-expansivity perturbation function $-T\left(\partial f_{2} / \partial T\right)_{p}$ as functions of temperature $T$ at various pressures $p$ : (a) to (c) $k_{1}=0.05 \mathrm{~K}^{-1}$; (d) to (f) $k_{1}=0.2 \mathrm{~K}^{-1}$. Curves: black, $p=1 \mathrm{MPa}$; blue, $p=100 \mathrm{MPa}$; red, $p=200 \mathrm{MPa}$; green, $p=300 \mathrm{MPa}$; purple, $p=400 \mathrm{MPa}$. 

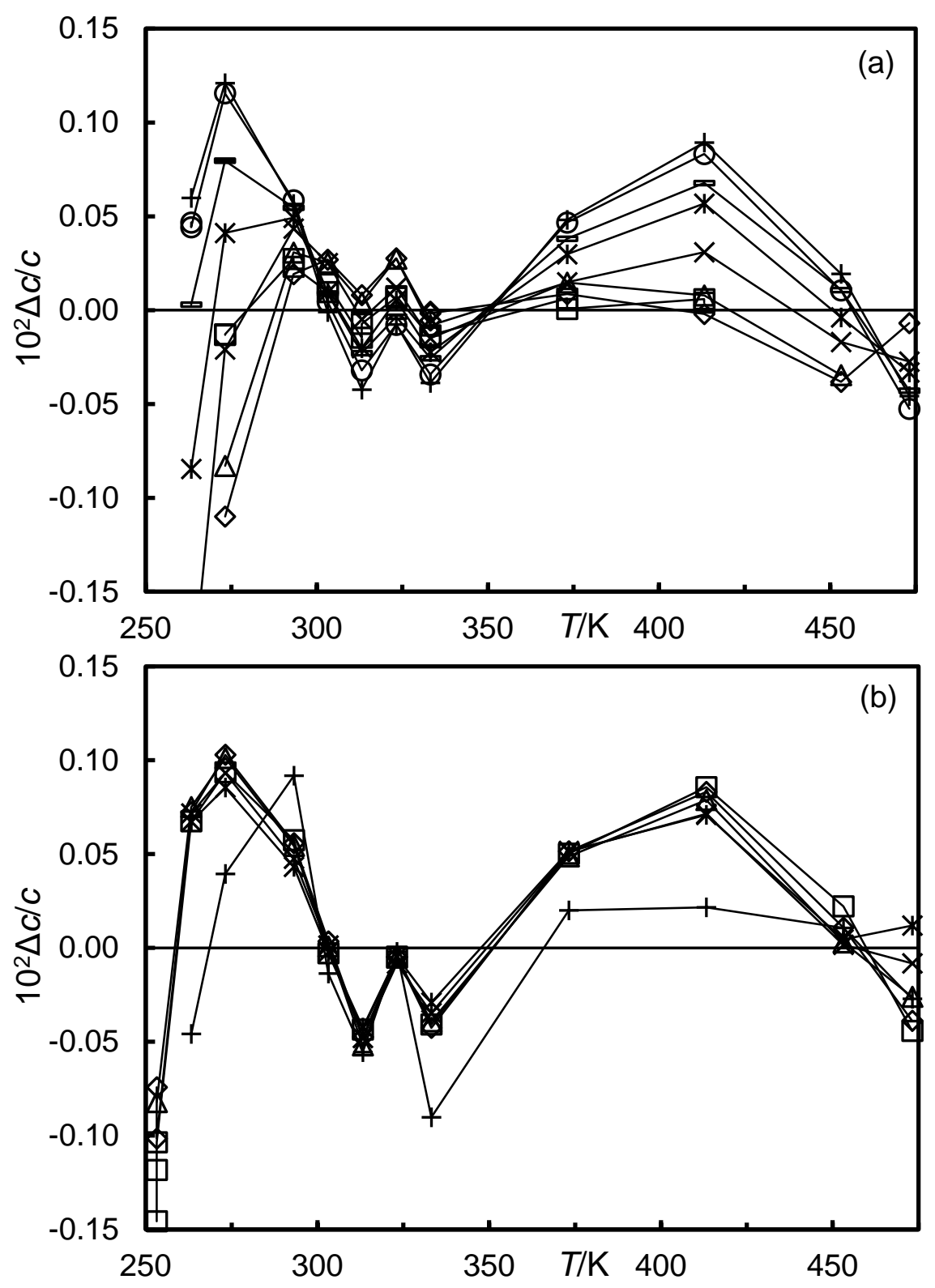

Figure 7. Relative deviations $\Delta c / c=\left(c_{\exp }-c_{\text {fit }}\right) / c_{\text {fit }}$ of experimental speeds of sound from the values $C_{\text {fit }}$ obtained from equations (15) and (16) with coefficients from Table 1 as a function of temperature $T$ for various nominal pressures $p$. (a) $\square, p=1 \mathrm{MPa} ; \diamond, p=25 \mathrm{MPa} ; \triangle, p=50$ $\mathrm{MPa} ; \times, p=75 \mathrm{MPa} ; *, p=100 \mathrm{MPa}$;,$p=125 \mathrm{MPa}$; O, $p=150 \mathrm{MPa}$; +, $175 \mathrm{MPa}$. (b) $\square, p=200 \mathrm{MPa} ; \diamond, p=225 \mathrm{MPa} ; \triangle, p=250 \mathrm{MPa} ; \times, p=275 \mathrm{MPa} ; *, p=300 \mathrm{MPa} ; \mathrm{O}$, $p=350 \mathrm{MPa} ;+, 400 \mathrm{MPa}$. Lines joining the points are intended only to guide the eye. 


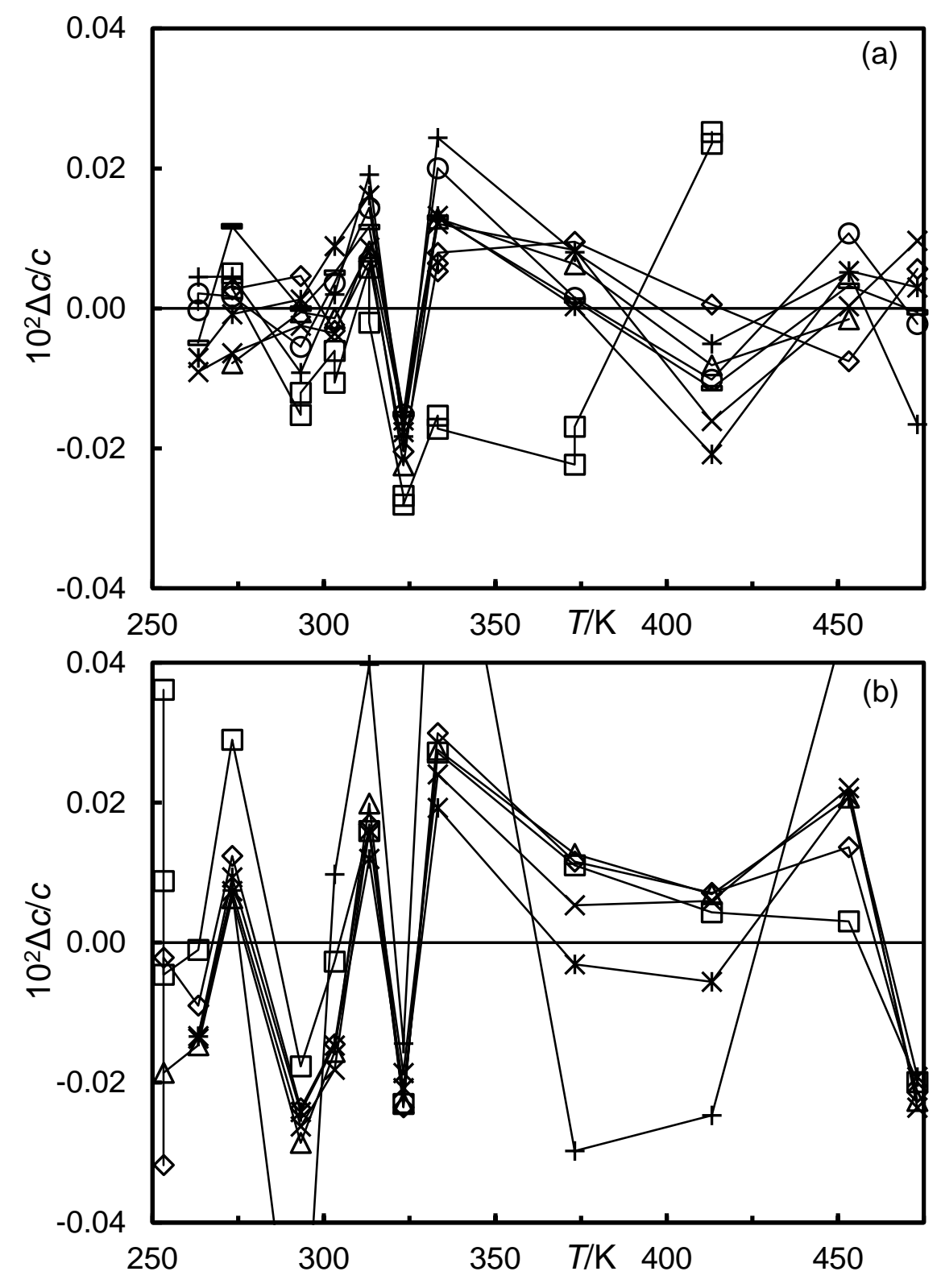

Figure 8. Relative deviations $\Delta c / c=\left(c_{\exp }-c_{\text {fit }}\right) / c_{\text {fit }}$ of experimental speeds of sound from the values $C_{\text {fit }}$ obtained from equations (15) and (16) with coefficients from Table 1 as a function of temperature $T$ for various nominal pressures $p$. (a) $\square, p=1 \mathrm{MPa} ; \diamond, p=25 \mathrm{MPa} ; \triangle, p=50$ $\mathrm{MPa} ; \times, p=75 \mathrm{MPa} ; *, p=100 \mathrm{MPa}$;,$p=125 \mathrm{MPa}$; O, $p=150 \mathrm{MPa}$; +, $175 \mathrm{MPa}$. (b) $\square, p=200 \mathrm{MPa} ; \diamond, p=225 \mathrm{MPa} ; \triangle, p=250 \mathrm{MPa} ; \times, p=275 \mathrm{MPa} ; *, p=300 \mathrm{MPa} ; \mathrm{O}$, $p=350 \mathrm{M} ` \mathrm{~Pa} ;+, 400 \mathrm{MPa}$. Lines joining the points are intended only to guide the eye. 


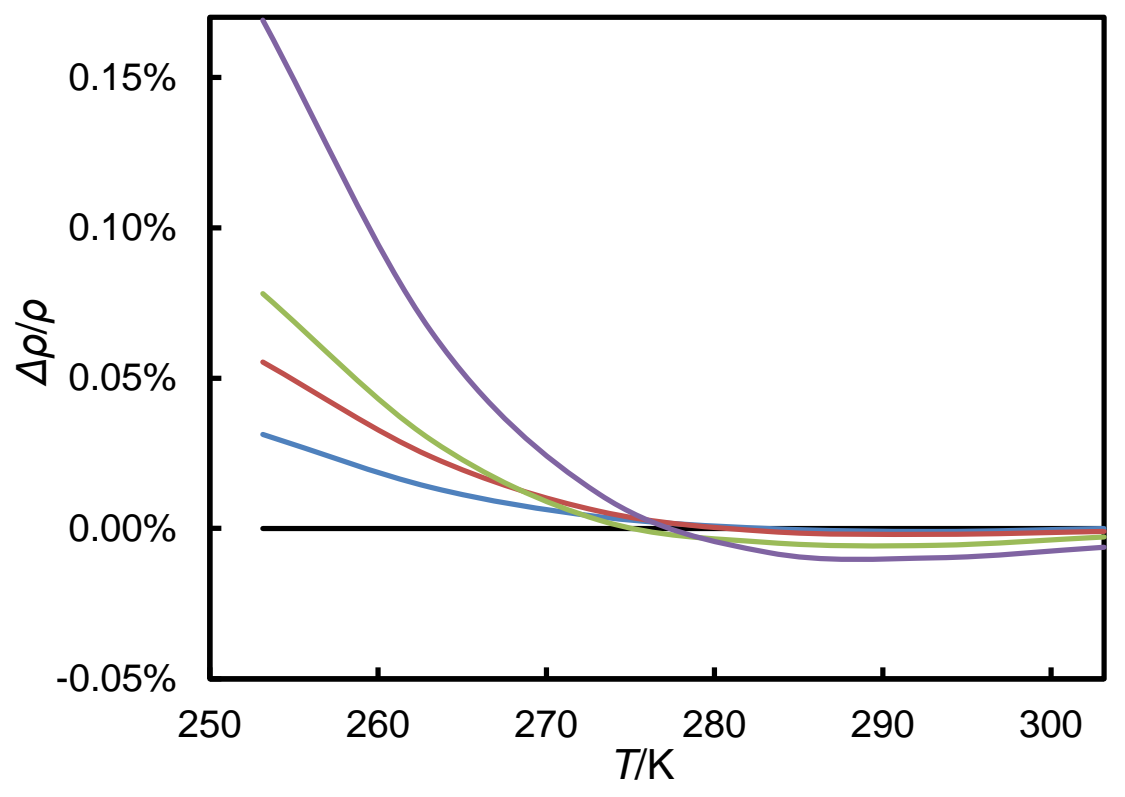

Figure 9. Differences $\Delta \rho=\left(\rho-\rho_{\mathrm{LT}}\right)$ between the densities $\rho$ of water obtained in this work and values $\rho_{\mathrm{LT}}$ reported by Lin and Trusler [8] as a function of temperature $T$ along isobars: black, $p=0.1 \mathrm{MPa}$; blue, $p=50 \mathrm{MPa}$; red, $p=100 \mathrm{MPa}$; green, $p=200 \mathrm{MPa}$; purple, $p=400 \mathrm{MPa}$. 


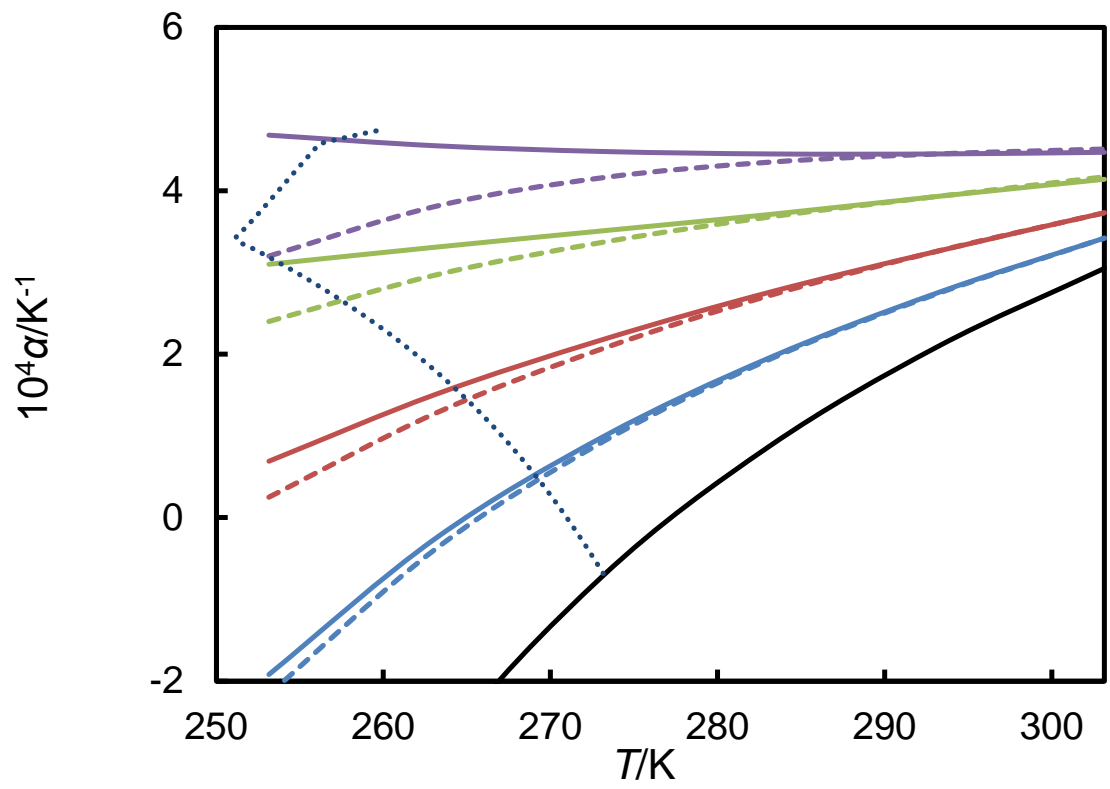

Figure 10. Isobaric expansivity $\alpha$ of water obtained by thermodynamic integration based on the present sound-speed correlation (solid curves) and the correlation of Lin and Trusler [8] (dashed curves) plotted as a function of temperature $T$ along isobars: black, $p=0.1 \mathrm{MPa}$; blue, $p=50 \mathrm{MPa}$; red, $p=100 \mathrm{MPa}$; green, $p=200 \mathrm{MPa}$; purple, $p=400 \mathrm{MPa}$. The dotted line gives the thermal expansivity of the saturated liquid on the melting curve according to the equation of state of Holten et al. [6]; the region to the left of this represents metastable liquid states. 


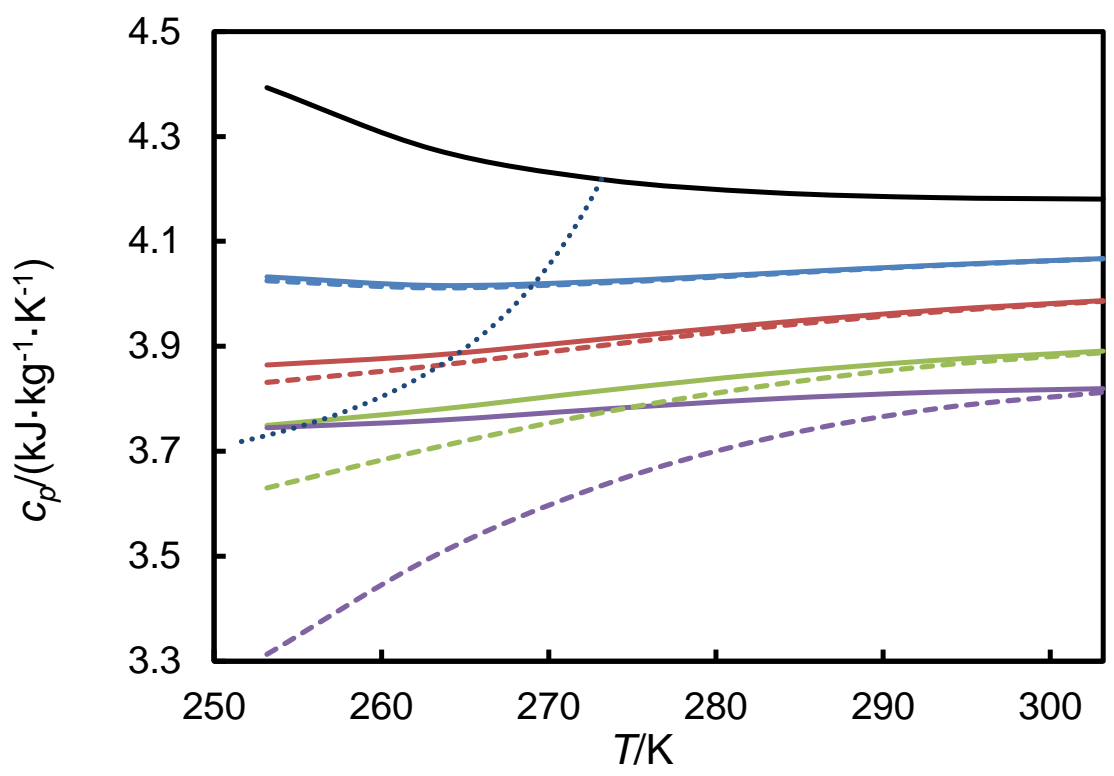

Figure 11. Isobaric specific heat capacity $c_{p}$ of water obtained by thermodynamic integration based on the present sound-speed correlation (solid curves) and the correlation of Lin and Trusler [8] (dashed curves) plotted as a function of temperature $T$ along isobars: black, $p=0.1 \mathrm{MPa}$; blue, $p=50 \mathrm{MPa}$; red, $p=100 \mathrm{MPa}$; green, $p=200 \mathrm{MPa}$; purple, $p=400 \mathrm{MPa}$. The dotted line gives the isobaric specific heat capacity of the saturated liquid on the melting curve according to the equation of state of Holten et al. [6]; the region above and to the left of this represents metastable liquid states. 